\title{
Can we rely on AGNP therapeutic targets also for LAI antipsychotics?
}

\author{
Sara Baldelli ${ }^{1}$, Emilio Clementi ${ }^{2,3}$ and Dario Cattaneo ${ }^{1}$
}

${ }^{1}$ Unit of Clinical Pharmacology, ASST Fatebenefratelli Sacco University Hospital, Milan, Italy

${ }^{2}$ Clinical Pharmacology Unit, Consiglio Nazionale delle Ricerche Institute of Neuroscience, Department of Biomedical and Clinical Sciences L. Sacco University Hospital, Università degli Studi di Milano, Milan, Italy

${ }^{3}$ E. Medea Scientific Institute, Bosisio Parini, Italy

Correspondence to: Sara Baldelli, Chem.D.

Unit of Clinical Pharmacology

ASST Fatebenefratelli Sacco University Hospital via GB Grassi 74, 20157 Milano, Italy;

E mail: sara.badelli@asst-fbf-sacco.it 
- 2 - 
Dear Editor,

We read with interest the updated AGNP Consensus Guidelines for Therapeutic Drug Monitoring (TDM) in Neuropsychopharmacology recently published in the journal. [1]. In this new version, the Authors have updated the available knowledge on the pharmacokinetics and pharmacogenetics of drugs used in psychiatry and neurology. In particular, the therapeutic reference ranges of 18 drugs have been revised, and novel reference ranges have been newly introduced for 28 drugs on the basis of recent published literature. In addition, the drug concentration-to-dose ratios have been extended to 120 neuropsychiatric drugs and introduced for 26 metabolites. Taken together, these updates have greatly improved the knowledge of the pharmacology of central nervous system, emphasizing at the same time the key role of TDM as a tool to individualize drug doses in psychiatric patients in the era of "precision medicine”.

We believe, however, that the updated guidelines have missed the important opportunity to face with, and to provide useful information on, the emerging issue of long-acting injectable (LAI) formulations of atypical antipsychotics. The role of LAI formulations - which have been developed as alternative to oral regimens, to sustain the release of the drug over a period of weeks, increasing the dosing interval time and improving patient adherence - is indeed growing [2-5].

Currently available LAI of second generation antipsychotics include aripiprazole, olanzapine, paliperidone and risperidone. According to the 2017 AGNP-TDM consensus guidelines, the level of recommendation for measuring concentrations in blood of these drugs is scored as " 1 ” (strongly recommended) or "2” (recommended). Therapeutic ranges have been proposed for each of these drugs, but it should be emphasized that these ranges are derived from studies dealing exclusively with drugs given as oral formulation. Therefore, their applicability in patients treated with the LAI formulations have not been established yet. 
This limitation opens the unaddressed question on how (and if) TDM should be applied also in patients shifted to or on maintenance therapy with LAI formulations of antipsychotics, as well exemplified below by our real life data.

In 2012 we have set up a TDM laboratory service for the assessment of plasma trough concentrations of antipsychotics by validated LC-MS/MS methods [6-7]. Looking in our database, we identified 81 patients on LAI antipsychotics (22 on aripiprazole, 28 on olanzapine and 31 on paliperidone) with multiple assessments (usually every two or four weeks). Interestingly, a high percentage of the samples resulted below the minimum effective antipsychotic concentrations suggested by the AGNP Guidelines whereas only a small percentage of the samples exceeding the upper threshold of the therapeutic ranges (Table 1). Despite the observation that patients on LAI just before the next injection show concentrations that were under the therapeutic range proposed for oral formulation, they were in any case clinically stable according to the physician who evaluated treatment efficacy by reduction of Brief Psychiatric Rating Scale (BPRS) and Positive and Negative Symptom Scale (PANSS).

These findings might raise the hypothesis that, also in the presence of the same drug, specific therapeutic reference ranges according to pharmaceutical formulations should be defined, especially for LAI, which present peculiar pharmacokinetic characteristics. We are of course aware that TDM data regarding these formulations are limited and that until new data are available, for instance regarding risperidone, the same therapeutic reference range has been proposed even if as underlined by the authors this may result in a gross simplification [8]. We are confident that, such data would increase in the near future allowing the inclusion of new tables dealing with LAI antipsychotics in the next AGNP guidelines. 


\section{References}

1. Hiemke C, Bergemann N, Clement et al. Consensus Guidelines for Therapeutic Drug Monitoring in Neuropsychopharmacology: Update 2017. Pharmacopsychiatry. 2017. doi: 10.1055/s-0043116492. Epub

2. Sreeraj VS, Shivakumar V, Rao NP, Venkatasubramanian G. A critical appraisal of long acting injectable antipsychotics: Translating research to clinics. Asian J Psychiatr. 2017; 28: 57-64.

3. Suzuki T. A further consideration on long-acting injectable versus oral antipsychotics in the treatment of schizophrenia: a narrative review and critical appraisal. Expert Opin Drug Deliv. 2016; 13: 253-64.

4. Biagi E, Capuzzi E, Colmegna F, et al. Long-Acting Injectable Antipsychotics in Schizophrenia: Literature Review and Practical Perspective, with a Focus on Aripiprazole Once-Monthly. Adv Ther. 2017; 34: 1036-1048.

5. Mohr P, Knytl P, Voráčková V, et al. Long-acting injectable antipsychotics for prevention and management of violent behaviour in psychotic patients. Int J Clin Pract. 2017; 71(9).

6. Baldelli S, Fucile S, Cattaneo D, et al. Development of a LC-MS/MS method for therapeutic drug monitoring of antidepressants and antipsychotics in human plasma. Ther Drug Monit 2011; 33: 528

7. Pozzi M, Cattaneo D, Baldelli S, et al. Therapeutic drug monitoring of second-generation antipsychotics in pediatric patients: an observational study in real-life settings. Eur J Clin Pharmacol. 2016; 72: 285-293.

8. Schoretsanitis G, Spina E, Hiemke C, et al. A systematic review and combined analysis of therapeutic drug monitoring studies for long-acting risperidone. Expert Rev Clin Pharmacol. 2017; 10: $965-981$ 
Table 1: Distribution of long acting injectable antipsychotic drug trough concentrations according to reference ranges of the updated AGNP guidelines

\begin{tabular}{lcccc}
\hline Drug & $\begin{array}{c}\text { Reference range } \\
(\mathbf{n g} / \mathbf{m L})\end{array}$ & $\begin{array}{c}\text { Samples } \\
\mathbf{( n )}\end{array}$ & $\begin{array}{c}\text { Sub-therapeutic } \\
\text { samples, n (\%) }\end{array}$ & $\begin{array}{c}\text { Supra-therapeutic } \\
\text { Samples, n (\%) }\end{array}$ \\
\hline Aripiprazole & $100-350$ & 129 & $47(36.4 \%)$ & $5(3.8 \%)$ \\
Olanzapine & $20-80$ & 352 & $206(58.5 \%)$ & $3(0.9 \%)$ \\
Paliperidone & $20-60$ & 430 & $138(34.2 \%)$ & $13(3 \%)$ \\
\hline
\end{tabular}

